\title{
Analysis on Postpartum Depression
}

\author{
Chunze Chen
}

\author{
Jinan NewChannel School, Jinan, Shandong Province, 250001, China \\ Corresponding author.Email: gaoming@cas-harbour.org
}

\begin{abstract}
Postpartum Depression (PPD) receives increasing attention from the public as well as in the academia. However, some of the facts about PPD are not as clear and simple as perceived by the public. Through an interdisciplinary literature review, this paper aims to help women at childbearing age, especially women in China, enhance their understanding of postpartum depression from the perspectives of physiological mechanism, social psychology, and psychodynamics. Critical views and indications of these risk factors are followed by each sector. Besides, treatment methods are presented briefly in the end.
\end{abstract}

Keywords: Postpartum depression, Definition and Diagnosis, Risk Factors and Pathophysiology, Impact on Children Development, Treatment

\section{INTRODUCTION}

Postpartum depression (PPD) affects $10 \%-15 \%$ of perinatal women worldwide [1]. In China, the prevalence is $16.3 \%$ in general population according to a meta-analysis in 2020 [2]. The ratio can vary largely in different demographic groups of age, region, education, socioeconomical status and other specific risk factors like chronic diseases. Especially, mothers over 35 years are most affected (31.25\% prevalence) comparing with other age groups due to a diversity of risk factors [3]. In 2021, 3-child policy has been issued in China and women are encouraged to give birth to 3 children, which not only extends childbearing age range but exposes women and their families to a wider scope of risks of PPD. Based on the huge potentially affected population, therefore, it is urgent for women at childbearing age in China to build a comprehensive understanding of PPD.

This essay examines the definition, diagnosis, epiology and risk factors, adverse effect, and treatment of PPD through studies both home and overseas. Suggestions are made in the end of the essay.

\section{DEFINITION AND DIAGNOSIS}

\subsection{Definition}

PPD is characterised by a series of major depressive symptoms with perinatal onset specifier.
The symptoms include diminished interest of pleasure in all or most of activities, insomnia or hypersomnia, psychomotor agitation or retardation, fatigue, feeling of loss of energy and recurrence of thought of death and Two of the essential diagnostic features are persisting depression mood and persisting anhedonia [4].

However, the definition of the specifier is not entirely consensus in different diagnostic criteria. In DSM-IV [5], the time frame is 4 weeks after delivery. In DSM-V [6], prenatal depression onset is included depression as many PPD episodes can begin during pregnancy.

Yet, the 4-week specifier is kept in the latest edition. This specifier is largely argued in academia as it may be overly strict for the diagnosis of PPD in practical terms. In ICD-10 [7], the onset time is within 6 weeks after delivery. Moreover, the time frame is prolonged to 12 months in the criteria of WHO [8]. In related studies, the screening for PPD is more often conducted in the 6th-8th weeks after delivery [9]. A new direction to define PPD is NIMH Research Domain Criteria (RDoC), which aims to integrate more underlying factors of genes, molecules, neural circuits, physiology, behaviour, and self-report instead of traditional nosological categories [10]. 


\subsection{Diagnosis}

In addition to the time specifier, 5 or more depressive symptoms are required to last for at least 2 weeks. Besides, maternal dysfunctional symptoms can be taken into consideration diagnosing PPD. Structural clinic interview and self-rating scales are commonly used approaches in the diagnosis of PPD [4].

Noteworthily, PPD must be distinguished from 'baby blues' and postpartum psychosis. Baby blues is also called postpartum blues. It has very similar symptoms with PPD but is milder, more transit and common mood disturbance than PPD. It usually occurs to $50 \%-80 \%$ of women and in 3 to 5 days after delivery. It can be recovered by 10 days. Suicidal ideation is not present in postpartum blues and no specific treatments are required. While postpartum psychosis is a psychiatric emergency. It occurs to $0.2 \%$ of women and can be seen as a manifestation of bipolar disorder. It has server risks of infanticide and suicide and therefore interventions are required [11,12,13]. Besides, Postpartum psychosis can be misdiagnosed as PPD. Wrong treatment and prescription can lead to increasing rate of suicide [14]

Self-rating scales as the effective and efficient instruments are commonly used in PPD screening and evaluation. Both scales specific for postpartum patients and scales for general population can be applied in screening. They can be used as alternatives or verification to improve the accuracy of PPD screening. Currently, the most used scale for PPD is Edinburgh Postnatal Depression Scale (EPDS). It is a 10 -item selfrating scale with high simplicity, sensitivity, and specificity. The cut-off score is 13 while $9 / 10$ is recommended for its Chinese version [15]. Another widely used scale for PPD is Postpartum Depression Screening Scale (PDSS), which has 35 items, and the highest score is 175 . Its cut-off score for mild and server postpartum depression is 76 and 98 respectively. Comparing with EPDS, it is considered better in terms sensitivity and specificity [16]. Among other scales screening depression in general population, Self-Rating Depression Scale (SDS) and The Patient Health Questionnaire 9 (PHQ-9) are widely used. Noteworthily, suicide ideation should receive special attention once it is reported regardless of the total score or scale type [4].

\section{RISK FACTORS AND PATHOPHYSIOLOGY}

A variety of risk factors for PPD have been identified and pathophysiology and theoretical frameworks are correspondingly developed. Although none of the risk factors can be seen as the ubiquitous cause of PPD, current findings suggest that PPD can be caused by the interaction of biological vulnerability and environmental factors. Reproductive Hormone changes, genetic factors, life stressors, and their interactions are identified to be associated with postpartum depression by abundant studies.

\subsection{Hormone changes}

\subsubsection{Reproductive Hormone changes}

Most reproductive hormone changes drastically during perinatal period and follow a general pattern that the concentration in the blood continues to increase in the 40 weeks of pregnancy, and then drops quickly after delivery [20]. Meanwhile, the effects of reproductive hormones are involved in almost all the pathophysiological mechanisms of depression [19]. However, there is no convincing evidence that compared with healthy women, PPD women have lower concentration, higher withdraw speed or greater reduction of reproductive hormones [19]. It is the fluctuation that triggers depressive symptoms and makes the perinatal period a high-incidence period for PPD [20]. Bloch et al. [21] stimulated such fluctuation on women with and without PPD history and found increasing depressive symptoms on women with PPD history. Another study provides evidence in the other way around [22]. It tried to identify the difference of cognitive style and personality between women with a recurrent episode of PPD and women without but failed to find any significant difference at week 6 after delivery. The result may indicate that there may be different PPD phenotypes and biomarker may play a role in distinguishing them.

\subsubsection{Other Hormone change}

Lactogenic hormones oxytocin also receives special attention in PPD studies as it modulates maternal behaviours and mood [23]. In addition, the increased corticotrophin releasing hormone $(\mathrm{CRH})$, adrenocorticotropic hormone (ACTH) and cortisol associated with hypothalamic-pituitary-adrenal (HPA) axis hyperactivity usually go back to normal at 12 weeks after delivery [24]. The changes also participate in the onset of depressive episode during perinatal period [25]. Besides, thyroid hormones may be another contributing factor to PPD as its dysfunction is one of the main risk factors of MDD although the association with PPD has not been clearly detected [19]. The aforementioned hormones are all under the regulation of reproductive hormones in the ways of binding globulins or gene expressions. Thus, they are potentially under the influence of reproductive hormones changes [26].

\subsection{Genetic factors}

Women with a family history had a 53 percent risk of developing postpartum depression, compared with 11.8 percent for women without a family history [27]. 
Current studies on epigenetics and behavioural genetics can reveal more about PPD etiologic features and its intergenerational transmission.

Hypermethylation of estragon-based DNA [28] and oxytocin receptor (OXTR) [29] is found to be associated with PPD. Also, the polymorphism of serotonin transporter (5-HTT), serotonin-transporterlinked polymorphic region (5-HTTLPR), catechol Omethyltransferase (COMT), monoamine oxidase-A (MAO-A) makes women more likely be influenced by hormonal changes [30]. The methylation can be associated with childcare stress, antenatal mood, lower level of education, childhood adversity and other environmental factors. Moreover, the foetus is extremely susceptible to the mother's painful emotions, and it might lead to an abnormal epigenetic expression of the offspring [31].

At the same time, children may acquire the behavioural and cognitive characteristics of mothers with PPD, and thus are more likely to develop depressive symptoms than others. The influence of PPD maternal behaviour on children will be discussed in the section of Impact of PPD on Children Development.

\subsection{Life stressors}

Women are exposed in drastically rising stressors in period of postpartum. Increased financial burden, decreased marriage satisfaction, ramification needed to cope with both welcomed or unwelcomed pregnancy, low social support received, childcare stress and bad infant temperament, can all be stressors associated with PPD [35]. In addition to the role of stress in methylation processes mentioned above, there are other frameworks developed to explain the connection between stressors and PPD.

\subsubsection{Cognitive theoretical frameworks}

Teering on the edge theory is developed by Beck. It described PPD as the feeling of losing control [33]. Attachment theory is proposed by Whiffen and Johnson that if mother and father cannot be securely attached during the anxiety-filled transition period to parenthood, it can lead to PPD [34]. Earthquake model is developed by Sichel and Driscoll. The analogy of 'an earthquake's used to explain PPD. The accumulated stressors can erupt as depression during postpartum period [35]. Interpersonal models are developed by Sullivan. He proposed that interpersonal experience shapes personality. The interpersonal relation is disturbed during the role transition to a new mother and stress and anxiety are generated. The security mechanism, including selective inattention, sublimination and dissociation, is activated to help reduce the stress [36]. Further, Beck explained that the discrepancy between the social support they desired and what they received influences depressive symptoms [37]. Meanwhile, low self-esteem personality is considered to have a medium effect size to PPD in meta-analysis [32].

\subsubsection{Sociocultural and Feminist perspectives}

Some investigators included the stressors outside the family and viewed PPD from sociocultural and feminist perspectives. Cultural-bound theory is proposed by Stern and Kruckman. They explained PPD as a western culture-bound illness as western culture tends to idealise motherhood. In addition, western women cannot enjoy the ritual social support and the recognition of being a vulnerable new mother as in cultures like East Asia [38]. Similarly, feminist theorists also suggested that PPD is associated with the idealised standard of motherhood imposed by medical and social political model [39].

\section{IMPACT OF PPD ON CHILDREN DEVELOPMENT}

Postnatal depressed mothers are less likely to take good care or give positive emotional response to children. Associations of PPD are found with the impairment to poorer physical development of infant, mother-infant relationship, and adverse child outcome through the pathways of genetics, physiological and neurological changes in children, maternal parenting, and stress in the social environment [40]. However, the associations found are not always clear and continuous in examined papers. Besides, age and gender of the impacted children can result in distinctive trajectories.

\subsection{Poorer physical development}

Studies show that the weight and height of 3-6month infant with postnatal depressed mothers are significantly lower than infants with well mothers [41]. But such influence might disappear after 12 months [42].

\subsection{Mother-infant relationship}

Compared with healthy mothers, postnatal depressed mothers showed lower amygdala activation and a faster decline in striatum activity on retest. As a result, PPD mothers respond less to both positive and negative emotions of their babies $[43,44]$. Further experiments showed no activation or low activation in the brain regions associated with postpartum depression when the mothers seeing faces of their babies crying or smiling. A baby's crying induces healthy mothers to adopt more active caregiving behaviours and a smiling face rewarded the mother. But mothers with postpartum depression lack such ability to empathize. As a result, there is less positive interaction between the mother and child $[45,46]$. 


\subsection{Adverse child outcome}

In late infancy, association between PPD and adverse emotional, behavioural, cognitive outcome is found but cannot be considered as predictors of the impairments. Infants at 19 months with postpartum depressive mother showed emotional features of less affective sharing, a lower rate of overall interaction, less concentration, and more negative responses and less sociability to a stranger [47]. In addition, behaviour difficulty like excessive temper tantrums and separation problems are also found in 18-month-old children [48]. Yet there are also studies indicate that postnatal mothers at 6 months is not a predictor of poorer cognitive and language development of the infants at 18 months [49].

In early childhood, puberty and later stages of life, such associations are found in studies, but they may require further examination. The incidence of ADHD syndrome is significantly higher than that of normal children [50]. Meta-analysis also shows that PPD is associated with lower IQ [51], but other investigators found no association with the IQ of 8-year-old children and PPD duration less than 1 year [52]. In addition, children with postnatal depressed mothers at school age showed significant lower school readiness [53]. But this result was found confined within boys [54] in a study and some of the studies even found girls with postnatal depressed mothers showed more pro-sociality [55].

Age and gender are variables of the impact of PPD on children. The two years after birth is regarded as a sensitive developmental period in children with postnatal depressed mothers [56]. Irreversible impairments are more likely to occur in this period [57]. However, as their age increased, the decrease of children's dependence on their mothers reduces the adverse effects of maternal depression on children's behaviour [58]. Meanwhile, male, and female children have distinctive trajectories. Boys are more influenced by the early onset of PPD while girls are more affected by interpersonal difficulties [59]. Besides, genetic factors play a greater role in the aetiology of PPD in girls than in boys [60].

\section{TREATMENT}

\subsection{Antidepressants}

Antidepressants have been found certain level of efficacy in alleviating depressive symptoms in PPD mother [61]. SSRIs antidepressants are used as first-line pharmacological treatment. Based on safety data from multiple studies, the risk of SSRIs for breastfeeding children is very low [62]. Sertraline and paroxetine are considered as the very safe pharmacological treatment as there is very low or nondetectable levels of medicine in infant sera and the absence of adverse infant effects [63]. However, the efficacy of antidepressants on improving maternal care is not evidently observed [64]. Besides, the efficacy of antidepressant can be challenged by hypogonadal status in postpartum period [65]. Therefore, it is not as efficacious as in nonpostpartum women and it may require longer therapeutic period [66].

\subsection{Hormone treatment}

Hormone therapies can be a remedy for antidepressant treatment to some extent. It has been proved that oxytocin can improve maternal behaviour and estriol can work as an adjunct treatment to improve the efficacy of antidepressants [23]. Yet, although oxytocin can improve mother-infant relationship and increase maternal sensitives [67], it cannot improve mood of PPD mothers or even worsen depressive symptoms [68]. In addition, long-term estrogen therapy can cause endometrial hyperplasia, which may increase the risk of endometrial cancer and thrombosis [69].

\subsection{Non-pharmacological interventions}

There are various non-pharmacological options for PPD. Psychotherapies are first-line interventions for PPD although antidepressant are more common used in clinic practices [70]. Psychotherapies are usually structural approaches delivered in 6-20 times on weekly basis [71]. Different psychotherapies focus differently. Cognitive behavioural therapy (CBT) is one of the most used psychotherapies. It usually focuses on the motherinfant relation and help to find the relation between emotion and behaviour [72]. Interpersonal psychotherapy is another commonly used intervention for PPD. It mainly focuses on four areas, including role transition, interpersonal disputes, grief, and interpersonal deficits [73] Systematic reviews shows that IPT can shorten the time of recovery [74]. Brief dynamic therapy (BDT) is a therapy based on psychoanalysis. It helps patients to better understand the unconscious and internal psychological conflicts. Other theoretical frameworks also provide perspectives for the treatment of PPD. For example, feminist theorists promote a more realistic concept for motherhood in social-political context. They encourage self-nurturance and expression of anger. Attachment theory investigators advocate marital therapy to facilitate emotion engagement between partners [75].

Noteworthily, the combined therapies of antidepressants and psychotherapies may not show significant advantages than monotherapies despite the differences of combination $[63,76]$. Moreover, neither of these treatments are found effective in long-term improvement of mother-child relationship [79].

Besides, there are other non-pharmacological interventions including dietary therapy, light therapy, repeated transcranial magnetic stimulation (rTMS), 
acupuncture and Chinese herbal medicine. They are proved to be effective in related studies, but lack of meta-analyses and systemic reviews.

\section{CONCLUSION}

Although giving birth to a child has long been regarded as a positive life event, the true experience of it may vary from person to person. For the large number of PPD affected mothers and their families, perinatal period can be a struggle. Maternal depressive symptoms hinder healthy infant development and family environment, yet the etiologies of PPD remain heterogenous and the risk factors can be mutually interacted. Therefore, a personalised treatment can be a better choice considering the different severity, focus, and breastfeeding plan of each family. However, the cost-effectiveness of accessible resources is rarely mentioned in domestic studies. Also, higher education level and a good command of perinatal knowledge are protective factors for PPD [2]. Thus, enhancement of related education can be a viable approach of prevention. A new direction is to take advantage of the Internet to spread related knowledge and conduct the screening and non-pharmacological interventions to minimalize the impact of PPD. For example, a team at Microsoft developed a model to identify mothers at risks of PPD on social media and achieved an accuracy of $80-83 \%$ [79]. After the implementation of 3-child policy in China, families are encouraged to have more children than before, which may increase Chinese women's exposure to PPD. The whole society need to give enough attention to the potential consequences and possible solutions of PPD before making family plans.

\section{REFERENCES}

[1] Gaynes BN, Gavin N, Meltzer-Brody S et al. Perinatal depression: Prevalence, screening accuracy, and screening outcomes: Summary. Evid Rep Technol Assess (Summ). 2005; (119): 1-8.

[2] Nisar A, Yin J, Waqas A et al. Prevalence of perinatal depression and its determinants in Mainland China: A systematic review and metaanalysis. Journal of Affective Disorders. 2020; 277: 1022-1037.

[3] Cheng XQ, Wang Y, Yang FX et al. Predisposing factors of postpartum depression and efficacy of psychological health care service. China Journal of Health. 2018; 26(11): 1612-1618.

[4] American Psychiatric Association, DSM-IV: Diagnostic and Statistical Manual of Mental Disorders, 4th Edition, Washington, DC: American Psychiatric Association, 1994.
[5] American Psychiatric Association. DSM-V: Diagnostic and Statistical Manual of Mental Disorders - DSM-5 5th Edition, Washington, DC: American Psychiatric Association Publishing, 2013.

[6] American Psychiatric Association. Diagnostic and statistical manual of mental disorders, Washington, DC: American Psychiatric Publishing, 2013: 186187.

[7] World Health Organization. The ICD-10 classification of mental and behavioural disorders: Clinical descriptions and diagnostic guidelines, Geneva: World Health Organization, 1992.

[8] World Health Organization. MhGAP intervention guide for mental, neurological and substance use disorders in non-specialized health settings: Mental health Gap Action Programme ( mhGAP), Geneva: World Health Organization, 2010.

[9] Li MM, Tang QF, Zhang GQ et al. Application of assessment scale for postnatal depression in Chinese papers. China Journal of Health. 2016; 30(6): 418-423.

[10] Melloni E, Benedetti F, Vai B et al. Not understanding others. The RdoC approach to Theory of mind and empathy deficits in Schizophrenia, Borderline Personality Disorder and Mood Disorders. Phenomenology and Mind. 2020; 2: 162-181.

[11] Buttner MM, O Hara MW, Watson D. The structure of women's mood in the early postpartum. Assessment. 2012; 19(2): 247-256.

[12] Cohen LSM, Wang BM, Nonacs RMP et al. Treatment of mood disorders during pregnancy and postpartum. The Psychiatric clinics of North America. 2010; 33(2): 273-293.

[13] Wisner K, Parry B, Piontek C. Clinical practice: Postpartum depression. New England Journal of Medicine. 2002; 347(3): 194-199.

[14] Rihmer Z, Gonda X, Serretti A. AntidepressantResistant depression and AntidepressantAssociated suicidal behaviour: The role of underlying bipolarity. Depression research and treatment. 2011; 2011(2090-1321): 906462906465 .

[15] Chen H, Bautista D, Ch'Ng YC et al. Screening for postnatal depression in Chinese-speaking women using the Hong Kong translated version of the Edinburgh Postnatal Depression Scale. AsiaPacific Psychiatry. 2013; 5(2): E64-E72.

[16] Liu RL, Liu M, Liao SL. Comparasion of two screening scales in screening postpartum 
depression. Chinese Journal of Modern Nursing. 2012; 18(19): 2274-2276.

[17] Skalkidou A, Hellgren C, Comasco E et al. Biological aspects of postpartum depression. Women's health (London, England). 2012; 8(6): 659-671.

[18] Henningsson S, Madsen KH, Pinborg A et al. Role of emotional processing in depressive responses to sex-hormone manipulation: A pharmacological fMRI study. Translational Psychiatry. 2015; 5(12): e688.

[19] Schiller CE, Meltzer-Brody S, Rubinow DR. The role of reproductive hormones in postpartum depression. Cns Spectrums. 2015; 20(1): 48-59.

[20] Maguire J, Mody I. GABAAR Plasticity during Pregnancy: Relevance to Postpartum Depression. Neuron (Cambridge, Mass.). 2008; 59(2): 207-213.

[21] Bloch M, Schmidt PJ, Danaceau M et al. Effects of gonadal steroids in women with a history of postpartum depression. The American journal of psychiatry. 2000; 157(6): 924-930.

[22] Jones L, Scott J, Cooper C et al. Cognitive style, personality and vulnerability to postnatal depression. British Journal of Psychiatry. 2010; 196(3): 200-205.

[23] Qiu TQ, Huang YJ, Gao J. Regulative effects of oxytocin on maternal behaviour of PPD mothers. Advances in Psychological Science. 2020; 28(3): 456-464.

[24] Mastorakos G, Ilias I. Maternal and fetal Hypothalamic-Pituitary-Adrenal axes during pregnancy and postpartum. Annals of the New York Academy of Sciences. 2003; 997(1): 136-149.

[25] Ross LE, Dennis C. The prevalence of postpartum depression among women with substance use, an abuse history, or chronic illness: A systematic review. Journal of women's health (Larchmont, N.Y. 2002). 2009; 18(4): 475-486.

[26] Ben-Rafael Z, Struass RJF, Arendash-Durand B et al. Changes in thyroid function tests and sex hormone binding globulin associated with treatment by gonadotropin. Fertility and Sterility. 1987; 48(2): 318-320.

[27] Kimmel M, Hess E, Roy PS et al. Family history, not lack of medication use, is associated with the development of postpartum depression in a highrisk sample. Archives of women's mental health. 2015; 18(1): 113-121.

[28] Kimmel M, Kaminsky Z, Payne JL. Biomarker or pathophysiology? The role of DNA methylation in postpartum depression. Epigenomics. 2013; 5(5): 473-475.

[29] King L, Robins S, Chen G et al. Perinatal depression and DNA methylation of oxytocinrelated genes: A study of mothers and their children. Hormones and Behavior. 2017; 96: 84-94.

[30] Jin CY, Ding MY. Prediction factors and Neurological Mechanism of Postpartum Depression. Advances in Psychological Science. 2017; 25(7): 1145

[31] Wei Y, Schatten H, Sun Q. Environmental epigenetic inheritance through gametes and implications for human reproduction. Human Reproduction Update. 2015; 21(2): 194-208.

[32] Beck CT. Predictors of postpartum depression: An update. Nursing Research. 2001; 50(5): 275-285.

[33] Beck CT. Teetering on the edge: A substantive theory of postpartum depression. Nursing Research. 1993; 42(1): 42-48.

[34] Whiffen VE, Johnson SM. An attachment theory framework for the treatment of childbearing depression. Clinical Psychology: Science and Practice. 1998; 5(4): 478-493.

[35] Sichel D, Driscoll JW. Women's moods: What every woman must know about hormones, the brain, and emotional health, New York: William Morrow New York, 1999.

[36] Sullivan HS. The interpersonal theory of psychiatry, New York: W. W. Norton, 1953.

[37] Beck CT. Theoretical perspectives of postpartum depression and their treatment implications. $\mathrm{MCN}$, the American journal of maternal child nursing. 2002; 27(5): 282-287.

[38] Stern G, Kruckman L. Multi-disciplinary perspectives on post-partum depression: An anthropological critique. Social Science \& Medicine. 1983; 17(15): 1027.

[39] Mauthner N. I. Towards a feminist understanding ofPostnatal depression'. Feminism \& Psychology. 1993; 3(3): 350-355.

[40] Goodman SH, Gotlib IH. Risk for psychopathology in the children of depressed mothers: A developmental model for understanding mechanisms of transmission. Psychological Review. 1999; 106(3): 458-490.

[41] Wojcicki JM, Holbrook K, Lustig RH et al. Chronic maternal depression is associated with reduced weight gain in latino infants from birth to 2 years of age. PLoS One. 2011; 6(2): e16737. 
[42] Grote V, Vik T, von Kries R et al. Maternal postnatal depression and child growth: A European cohort study. BMC Pediatrics. 2010; 10(1): 14.

[43] Silverman ME, Loudon H, Liu X et al. The neural processing of negative emotion postpartum: A preliminary study of amygdala function in postpartum depression. Archives of women's mental health. 2011; 14(4): 355-359.

[44] Moses-Kolko EL, Fraser D, Wisner KL et al. Rapid habituation of ventral striatal response to reward receipt in postpartum depression. Biological Psychiatry. 2011; 70(4): 395-399.

[45] Laurent HK, Ablow JC. A cry in the dark: Depressed mothers show reduced neural activation to their own infant's cry. Social Cognitive and Affective Neuroscience. 2012; 7(2): 125-134.

[46] Laurent HK, Ablow JC. A face a mother could love: Depression-related maternal neural responses to infant emotion faces. Social Neuroscience. 2013; 8(3): 228-239.

[47] Stein A, Gath DH, Bucher J et al. The Relationship between Post-natal Depression and Mother-Child Interaction. British Journal of Psychiatry. 1991; 158(1): 46-52.

[48] Murray L. The Impact of Postnatal Depression on Infant Development. Journal of Child Psychology and Psychiatry. 1992; 33(3): 543-561.

[49] Piteo AM, Yelland LN, Makrides M. Does maternal depression predict developmental outcome in 18 month old infants? Early Human Development. 2012; 88(8): 651-655.

[50] Chronis AM, Lahey BB, Pelham WE et al. Maternal depression and early positive parenting predict future conduct problems in young children with Attention-Deficit/Hyperactivity disorder. Developmental Psychology. 2007; 43(1): 70-82.

[51] Sui G, Pan B, Liu G et al. The Long-Term Effects of Maternal Postnatal Depression on a Child's Intelligence Quotient: A Meta-Analysis of Prospective Cohort Studies Based on 974 Cases. The journal of clinical psychiatry. 2016; 77(11): e1474-e1482.

[52] Evans J, Melotti R, Heron J et al. The timing of maternal depressive symptoms and child cognitive development: A longitudinal study. Journal of Child Psychology and Psychiatry. 2012; 53(6): 632-640.

[53] Barker ED, Jaffee SR, Uher $R$ et al. The contribution of prenatal and postnatal maternal anxiety and depression to child maladjustment. Depression and Anxiety. 2011; 28(8): 696-702.
[54] Sinclair D, Murray L, Stein A et al. Teacher reports of adjustment to school of postnatally depressed and well mothers' children. The British Journal of Psychiatry. 1998; 172: 58-63.

[55] Murray L, Cooper PJ. Postpartum depression and child development. Psychological Medicine. 1997; 27(2): 253-260.

[56] Bagner DM, Pettit JW, Lewinsohn PM et al. Effect of maternal depression on child behavior: A sensitive period? Journal of the American Academy of Child $\backslash \&$ Adolescent Psychiatry. 2010; 49(7): 699-707.

[57] Hay DF, Pawlby S, Waters CS et al. Antepartum and postpartum exposure to maternal depression: Different effects on different adolescent outcomes. Journal of Child Psychology and Psychiatry. 2008; 49(10): 1079-1088.

[58] Lovejoy MC, Graczyk PA, O'Hare E et al. Maternal depression and parenting behavior: A meta-analytic review. Clinical Psychology Review. 2000; 20(5): 561-592.

[59] Hammen C, Brennan PA, Keenan-Miller D. Patterns of adolescent depression to age 20: The role of maternal depression and youth interpersonal dysfunction. Journal of Abnormal Child Psychology. 2008; 36(8): 1189-1198.

[60] Kendler KS, Gardner CO, Neale MC et al. Genetic risk factors for major depression in men and women: Similar or different heritabilities and same or partly distinct genes? Psychological Medicine. 2001; 31(4): 605-616.

[61] Patel M, Bailey RK, Jabeen S et al. Postpartum depression: A review. Journal of Health Care for the Poor and Underserved. 2012; 23(2): 534-542.

[62] Orsolini L, Bellantuono C. Serotonin reuptake inhibitors and breastfeeding: A systematic review. Human psychopharmacology. 2015; 30(1): 4-20.

[63] O'Hara MW, Mccabe JE. Postpartum depression: Current status and future directions. Annual Review of Clinical Psychology. 2013; 9(1): 379407.

[64] Brummelte S, Galea LAM. Postpartum depression: Etiology, treatment and consequences for maternal care. Hormones and Behavior. 2016; 77: 153-166.

[65] Bloch M, Daly RC, Rubinow DR. Endocrine factors in the etiology of postpartum depression. Comprehensive Psychiatry. 2003; 44(3): 234-246.

[66] Sharma V, Sommerdyk C. Are antidepressants effective in the treatment of postpartum depression? 
A systematic review. Primary care companion for CNS disorders. 2013; 15(6).

[67] Feldman R, Zagoory-Sharon O, Weisman O et al. Sensitive parenting is associated with plasma oxytocin and polymorphisms in the OXTR and CD38 genes. Biological psychiatry (1969). 2012; 72(3): 175-181.

[68] Mah BL, Van Ijzendoorn MH, Smith R et al. Oxytocin in postnatally depressed mothers: Its influence on mood and expressed emotion. Progress in Neuro-Psychopharmacology \& Biological Psychiatry. 2013; 40: 267-272.

[69] Dennis C, Ross LE, Herxheimer A. Oestrogens and progestins for preventing and treating postpartum depression. Cochrane Database of Systematic Reviews. 2008; 2(4): D1690.

[70] Pearlstein TB, Zlotnick C, Battle CL et al. Patient choice of treatment for postpartum depression: A pilot study. Archives of women's mental health. 2006; 9(6): 303-308.

[71] Bobo WV, Yawn BP. Concise review for physicians and other clinicians: Postpartum depression. Mayo Clinic Proceedings. 2014; 89(6): 835-844.

[72] Sockol LE. A systematic review of the efficacy of cognitive behavioral therapy for treating and preventing perinatal depression. Journal of Affective Disorders. 2015; 177: 7-21.

[73] Beck CT. Theoretical perspectives of postpartum depression and their treatment implications. MCN, the American journal of maternal child nursing. 2002; 27(5): 282-287.

[74] Miniati M, Callari A, Calugi S et al. Interpersonal psychotherapy for postpartum depression: A systematic review. Archives of women's mental health. 2014; 17(4): 257-268.

[75] Kornaros K. Treating postpartum emotional distress by a short-term psychodynamic infantparent intervention integrated with Child Health Center care. Dept of Women's and Children's Health. 2020

[76] Song CF, Zhang E, Xia Z. The therapeutic effects of sertraline with cognitive-behavioral therapy intervention on postpartum depression. Journal of Clinical Psychiatry. 2013; 23(2): 112-114.

[77] Appleby L, Warner R, Whitton A et al. A controlled study of fluoxetine and cognitivebehavioural counselling in the treatment of postnatal depression. British Medical Journal. 1997; 314(7085): 932-936.
[78] Huang R, Yang D, Lei B et al. The short- and long-term effectiveness of mother-infant psychotherapy on postpartum depression: A systematic review and meta-analysis. Journal of Affective Disorders. 2020; 260: 670-679.

[79] De Choudhury M, Counts S, Horvitz E. Predicting postpartum changes in emotion and behavior via social media. Proceedings of the SIGCHI Conference on Human Factors in Computing Systems. New York: ACM. 